\title{
Visualization of Guideline-Based Decision Support for the Management of Pressure Ulcers in Nursing Homes
}

\author{
Abir ABDELLATIF ${ }^{\mathrm{a}, \mathrm{b}, \mathrm{c}, 1}$, Jacques BOUAUD ${ }^{\mathrm{d}, \mathrm{a}}$, \\ Joël BELMIN ${ }^{\mathrm{e}, \mathrm{b}}$ and Brigitte SEROUSSI ${ }^{\mathrm{a}, \mathrm{f}}$ \\ a Sorbonne Université, Université Sorbonne Paris Nord, Inserm, UMR S_1142, \\ LIMICS, Paris, France \\ ${ }^{\mathrm{b}}$ AP-HP, Hôpital Charles-Foix, Ivry-sur-Seine, France \\ ${ }^{\mathrm{c}}$ Teranga Software, Paris, France \\ d AP-HP, DRCI, Paris, France \\ e Sorbonne Université, Paris, France \\ ${ }^{\mathrm{f}}$ AP-HP, Hôpital Tenon, Paris, France
}

\begin{abstract}
Though a preventable risk, the management of pressure ulcers (PUs) in nursing homes is not satisfactory due to inadequate prevention and complex care plans. PUs early detection and wound assessment require to know the patient condition and risk factors and to have a good knowledge of best practices. We built a guideline-based clinical decision support system (CDSS) for the prevention, the assessment, and the management of PUs. Clinical practice guidelines have been modeled as decision trees and formalized as IF-THEN rules to be triggered by electronic health record (EHR) data. From PU assessment yielded by the CDSS, we propose a synthetic visualization of $\mathrm{PU}$ current and previous stages as a gauge that illustrates the different stages of PU continuous evolution. This allows to display PU current and previous stages to inform health care professionals of PU updated assessment and support their evaluation of previously delivered care efficiency. The CDSS will be integrated in NETSoins nursing homes EHR where gauges for several health problems constitute a patient dashboard.
\end{abstract}

Keywords. Clinical decision support system, clinical practice guidelines, information display, nursing homes, pressure ulcer, geriatrics

\section{Introduction}

Pressure ulcers (PUs) are injuries to the skin and underlying tissues. These ulcers are painful and significantly reduce a person's quality of life. PUs are expensive to manage and impact negatively the achievement of cost-effective and efficient care delivery. In most countries, PUs are thus considered as key clinical care quality indicators in care facilities. Yet, physically limited or bedridden elderly in NHs frequently suffer from PUs, and best practices for prevention and treatment of PUs may not be systematically applied

\footnotetext{
${ }^{1}$ Corresponding Author, Abir Abdellatif, Hôpital Charles-Foix, 7 avenue de la République, 94200 Ivrysur-Seine, France. E-mail: abir.abdellatif@gmail.com
} 
[1]. Incidence studies report PU figures between $6.2 \%$ and $8.8 \%$ in hospitalized geriatric patients, and even higher figures (between $11.9 \%$ and $23.2 \%$ ) in NHs [2].

Clinical practice guidelines (CPGs) are narrative recommendations about the care of patients with specific conditions. They have the potential to reduce unwarranted practice variation and improve healthcare quality and safety. Guideline-based clinical decision support systems (CDSSs) that provide patient-specific recommended care protocols have shown to positively impact patient clinical outcomes, e.g., reduction of PU incidence [3], and to support nurses in improving PU assessment documentation [4].

However, few CDSS are routinely used in NHs. One reason is that NHs have been slow to adopt health information technology tools, e.g., computerized medical records. Another reason could be that currently developed CDSSs do not meet NH users' expectations. For instance, alert fatigue and alert overrides may question the classic display of recommendations. In a previous work [5], we have proposed to implement gauges gathered in dashboards to represent guideline-based recommendations for the management of $\mathrm{NH}$ resident malnutrition. This paper presents the application of this method to the prevention and management of PUs. This work has been conducted within the NETSmart project that aims at developing guideline-based CDS modules to enrich the NETSoins EHR developed by Teranga Software in France ${ }^{2}$.

\section{Material and Methods}

\subsection{Pressure ulcer risk assessment, prevention, and management}

We chose to implement the national CPGs developed by the French agency for healthcare quality "Haute Autorité de Santé" for the prevention and the management of pressure ulcers. We used Shiffman's method [6] to identify both decision and action variables and translate guideline knowledge into a decision tree, finally rewritten as human-readable IF-THEN decision rules. The assessment and management of PUs requires a multidisciplinary approach. Nurses are the main actors in pressure ulcer care management. However, physicians are also involved in the therapeutic management and follow-up, especially when classic PU therapeutic protocols are not efficient.

PU management differs according to the existence of a PU wound and PU risk factors. The wound is clinically established and documented by nurses. CPGs recommend to consider ten risk factors: immobilization with limited ability of reposition $>3$ hours, return from intensive care $<24$ hours, malnutrition, dehydration, urinary or fecal incontinence, antecedent of PU, hypotension, perfusion, consciousness disorders, nervous system diseases.

When there is a wound, both the wound and PU risk factors should be assessed: the treatment includes the therapeutic management of the wound (including the management of pain, flushing, exudate, and necrotic tissues) and monitoring of risk factors. When there is no PU wound, then risk factors should be assessed and controlled, the skin should be inspected daily to identify the presence of erythema. PU management is organized in two main steps: PU risk assessment and prevention protocol, wound management and follow-up. Figure 1 displays the guideline-based decision tree of PU prevention, management, and follow up.

\footnotetext{
${ }^{2}$ This research is partially funded by ANRT CIFRE Grant n ${ }^{\circ} 2018 / 0307$ for AA.
} 


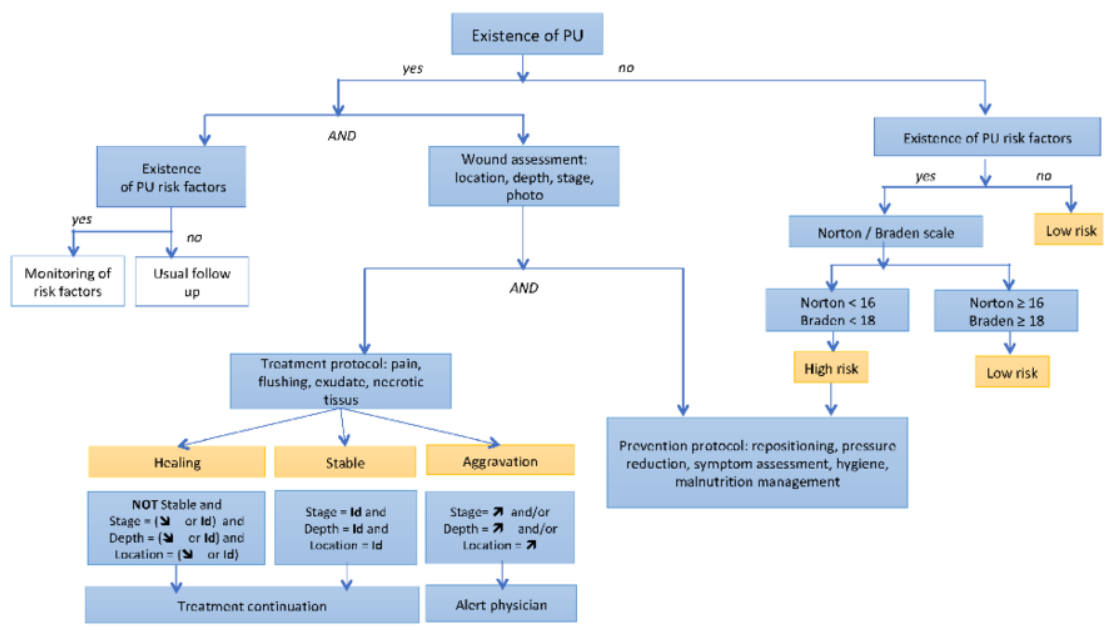

Figure 1. Decision tree for PU assessment, prevention, and management (Id means no modification).

- PU risk assessment and prevention: this applies when there is no PU wound. Daily, the CDSS evaluate all PU risk factors from EHR data. If there is no risk factor then PU risk is computed as low. If there is at least one PU risk factor, then PU assessment scales should be completed by nurses (i.e. Norton or Braden). According to the PU scale values, PU risk is computed as high (Braden $<18 /$ Norton $<16$ ) or low (Braden $\geq 18 /$ Norton $\geq 16$ ). When PU risk $=$ low, the level of the risk is displayed in the system interface, and no particular preventive action is recommended. When PU risk = high, then PU prevention protocol (including repositioning every two hours, pressure reduction, symptom assessment, hygiene, malnutrition management) is displayed along with the level of the risk. Recommendations to monitor detected PU risk factors are provided.

- PU wound management and follow-up: this applies when there is at least one PU wound. In this case, nurses have to document the wound (wound stage, depth, location) and it is recommended that they take a picture of the wound to be included in the EHR. Once the stage is established, both the prevention and the stage-specific therapeutic protocols are triggered. The therapeutic protocol is provided according to the patient symptoms recorded in his/her EHR (e.g., when the patient suffers from pain, analgesics are recommended). Reassessment of PU wound and risk factors is performed on a daily basis. According to the wound evolution (healing, stable, aggravation), CPGs provide follow-up recommendations including repositioning, fluid therapy, or alerting the physician in case of aggravation despite the implementation of the therapeutic protocol and the correction of risk factors. 


\subsection{CDSS visualization of alerts and recommendations}

As already done with malnutrition [5], we propose to display PU assessment by using a gauge (see Figure 2). When there is no PU wound, the gauge uses icons to indicate the risk of PU (low risk in green and high risk in red). When there is a PU wound, the display relies on a graphical drawing to illustrate the different stages of PU continuous evolution, from stage I, characterized by superficial reddening of the skin to stage IV where PUs are the deepest, extending into muscle, tendon, ligament, cartilage, or bone.

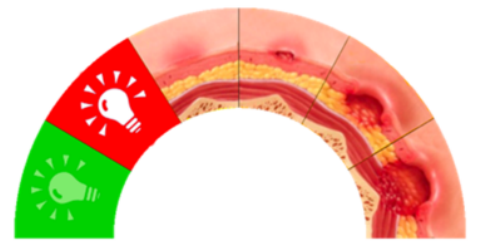

Figure 2. Display of PU assessment with the stepwise evolution from low risk to stage IV.

A pilot evaluation of gauges used as graphical user interfaces to present patient data and the display of recommendations has been made using three focus groups on a sample of care practitioners. Each focus groups lasted 30 minutes and was made of three steps: (i) presentation of the CDSS functionalities and graphical interfaces on a simulated clinical case, (ii) open discussion to let participants share their opinions on advantages and drawbacks of the presented interfaces, (iii) qualitative user assessment through questionnaires derived from the USE questionnaire ${ }^{3}$.

\section{Results}

To illustrate the CDSS processing, we considered the case of a 74-year-old patient with moderate Alzheimer disease. At $\mathrm{NH}$ admission in March 2020 , weight $=75 \mathrm{~kg}$, height $=1.80 \mathrm{~m}, \mathrm{BMI}=23 \mathrm{~kg} / \mathrm{m} 2, \mathrm{Alb}=40 \mathrm{~g} / \mathrm{l}$, and $\mathrm{MNA}=22$. Three weeks later, on April $3^{\text {rd }}, 2020$, new measures (weight $=70.5 \mathrm{~kg}$, Braden score $=8$ for a reddening of the skin located at the heel) triggered (i) a moderate malnutrition alert, and (2) a high risk PU alert, along with the recommendations to manage the two health problems. About two weeks later, on April $15^{\text {th }}$, heel pressure ulcer rapidly worsened to stage I in a context of broncho-pneumopathy associated with a depression episode, and malnutrition status became severe. On April $18^{\text {th }}$, we observed a worsening of the wound with loss of superficial skin staged as a stage II PU. The two leftmost gauges in the EHR user interface displayed in Figure 3 represent malnutrition status and PU status in their current (black cursor) and previous (grey cursor) states thus providing information about the evolution.

The display of EHR interfaces as gauges and dashboards was assessed by a sample of 16 care practitioners (six geriatricians, six nurses, two care assistants, one dietician, and one psychometrician). Most of them considered this visualization modality was useful or very useful $(94 \%)$, easy or very easy to use $(63 \%)$, easy or very easy to learn $(88 \%)$, and they were globally $88 \%$ to think they could be satisfied with it.

\footnotetext{
${ }^{3} \mathrm{https://garyperlman.com/quest/quest.cgi?form=USE}$
} 


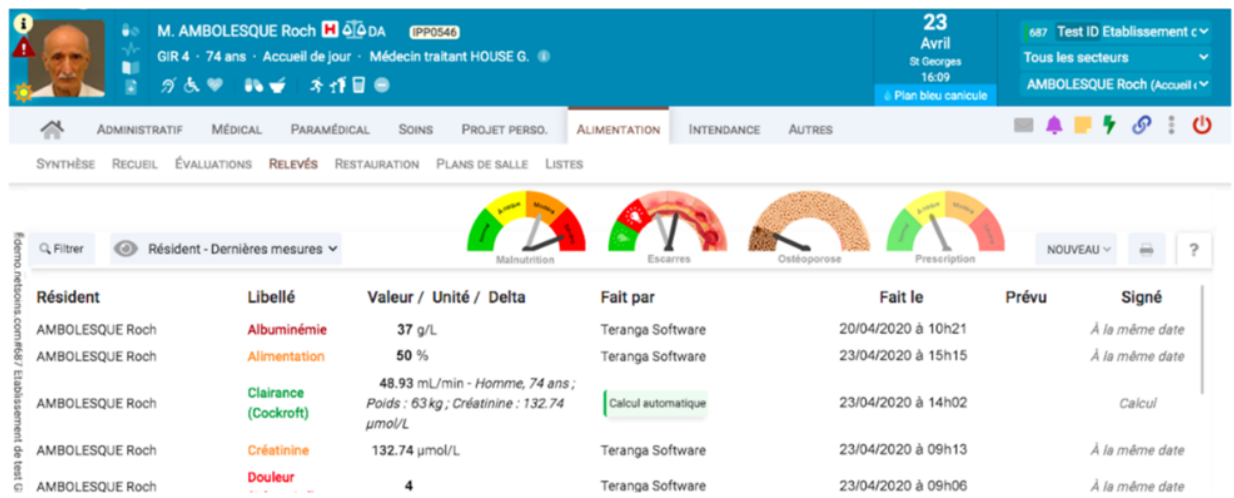

Figure 3. Dashboard visualization included in the NETSoins EHR interface.

\section{Discussion and Conclusions}

We are developing a CDSS to assist healthcare professionals in improving quality of care in NHs by supporting the management of common critical conditions like PUs, malnutrition, osteoporosis, and polymedication. Because of PU assessment difficulties and of the need for well-coordinated and guideline-based care plans, we proposed a DS module to evaluate patient PU risk or severity to provide management and follow-up recommendations, similar to the malnutrition DS module [5]. The extra-value of PU stage representation is to provide a graphical illustration. It is currently the user's responsibility to match the patient's PU to the appropriate stage illustration, and AI techniques would automatically classify PU stage from the skin picture currently taken and included within the resident EHR. Such graphical proposal has been assessed in focus groups with regular users of NH EHR systems and well accepted despite some training guidance seems necessary (only $63 \%$ found the visualization easy or very easy to use). However further work is needed to test the CDSS under real conditions.

\section{References}

[1] White EM, Aiken LH, McHugh MD. Registered Nurse Burnout, Job Dissatisfaction, and Missed Care in Nursing Homes. J Am Geriatr Soc. 2019;67(10):2065-2071.

[2] Beeckman D, Clays E, Van Hecke A, Vanderwee K, Schoonhoven L, Verhaeghe S. A multi-faceted tailored strategy to implement an electronic clinical decision support system for pressure ulcer prevention in nursing homes: a two-armed randomized controlled trial. Int J Nurs Stud. 2013;50(4):475-486.

[3] Olsho LE, Spector WD, Williams CS, et al. Evaluation of AHRQ's on-time pressure ulcer prevention program: a facilitator-assisted clinical decision support intervention for nursing homes. Med Care. 2014;52(3):258-266.

[4] Fossum M, Ehnfors M, Svensson E, Hansen LM, Ehrenberg A. Effects of a computerized decision support system on care planning for pressure ulcers and malnutrition in nursing homes: an intervention study. Int J Med Inform. 2013;82(10):911-921.

[5] Abdellatif A, Bouaud J, Belmin J, Seroussi B. Guideline-Based Decision Support System for Nursing Homes: A Case Study with the Management of Malnutrition. Stud Health Technol Inform. 2020;272:296299.

[6] Shiffman RN, Michel G, Essaihi A, Thornquist E. Bridging the guideline implementation gap: a systematic, document-centered approach to guideline implementation. J Am Med Inform Assoc. 2004;11(5):418-426. 